\title{
$A$-Harmonic operator in the Dirac system
}

\section{Fengfeng Sun and Shuhong Chen*}

"Correspondence: shiny0320@163.com

School of Mathematics and Statistics, Minnan Normal University, Zhangzhou, Fujian 363000, China

\section{Abstract}

In this paper, we show how an A-harmonic operator arises from A-Dirac systems under natural growth condition. By the method of removable singularities for solutions to A-Dirac systems with natural growth conditions, we establish the fact that A-harmonic equations are the real part of the corresponding A-Dirac systems.

Keywords: A-harmonic operator; A-Dirac system; Caccioppoli estimate; natural growth condition

\section{Introduction}

In this paper, we derive the relation between an $A$-harmonic operator and $A$-Dirac systems under natural growth condition. The equation defined by an $A$-harmonic operator in the current paper is

$$
-\operatorname{div} A(x, \nabla u)=f(x, \nabla u),
$$

where

$$
A(x, \xi): \Omega \times \mathbb{R}^{n} \rightarrow \mathbb{R}^{n}
$$

$x \rightarrow A(x, \xi)$ is measurable for all $\xi$, and $\xi \rightarrow A(x, \xi)$ is continuous for a.e. $x \in \Omega$. Further assume that $A(x, \xi)$ satisfies the following structure growth conditions for some constants $a>0$ and $\lambda>0$, with exponent $p>1$ :

$$
\langle A(x, \xi), \xi\rangle \geq \lambda|\xi|^{p}, \quad|A(x, \xi)| \leq a|\xi|^{p-1}
$$

and $f(x, \xi)$ satisfies the following natural growth condition for certain constants $C_{1}$ and $C_{2}$ :

$$
f(x, \nabla u) \leq C_{1}|\nabla u|^{p}+C_{2}, \quad|u|<M<+\infty \text { and } \lambda>C_{1} M .
$$

The exponent $p$ represents an exponent throughout the paper.

Definition 1.1 We call the function $u \in W_{\text {loc }}^{1, p}(\Omega) \cap L^{\infty}(\Omega)$ a weak solution to (1.1) under structure growth conditions (1.3) and (1.4) if the equality

$$
\int_{\Omega}\langle A(x, \nabla u), \nabla \phi\rangle=-\int_{\Omega}\langle f(x, \nabla u), \phi\rangle
$$

holds for all $\phi \in W^{1, p}(\Omega)$ with compact support.

\section{Springer}

(02013 Sun and Chen; licensee Springer. This is an Open Access article distributed under the terms of the Creative Commons Attribution License (http://creativecommons.org/licenses/by/2.0), which permits unrestricted use, distribution, and reproduction in any medium, provided the original work is properly cited. 
The $A$-Dirac systems in the paper are of the form

$$
-D \tilde{A}(x, D u)=f(x, D u)
$$

The main purpose of this paper is to show that under natural growth condition, an equation defined by an $A$-harmonic operator is the real part of the corresponding $A$-Dirac system. The properties of the Dirac operator are stated in Section 2. Further discussion on nonlinear Dirac equations can be found in [1-9] and references therein.

In order to obtain the desired results, we prove that under natural growth condition, a result concerning removable singularities for equations defined by an $A$-harmonic operator satisfying the Lipschitz condition or of bounded mean oscillation extends to Cliffordvalued solutions to corresponding Dirac equations.

The method of removability theorems was introduced in [1] for monogenic functions with modulus of continuity $\omega(r)$, in which the sets of $r^{n} \omega(r)$-Hausdorff measure are removable. The results were extended to Hölder continuous analytic functions by Kaufman and Wu in [10]. Then, the sets satisfying a certain geometric condition related to Minkowski dimension were shown to be removable for $A$-harmonic functions in Hölder and bounded mean oscillation classes in [11]. In the case of Hölder continuity, this was sharpened in [12] to a precise condition for removable sets for $A$-harmonic functions in terms of Hausdorff dimension. In [3], the sets satisfying a generalized Minkowski-type inequality were removable for solutions to the $A$-Dirac equation which satisfy a certain oscillation condition.

In this paper, we show that the above results even hold for inhomogeneity equations defined by an $A$-harmonic operator and $A$-Dirac systems, and we establish that under natural growth condition, the removable theorem holds for solutions to the corresponding $A$-Dirac equations and the main result can be stated as follows.

Theorem 1.2 Let $E$ be a relatively closed subset of $\Omega$. Suppose that $u \in L_{\mathrm{loc}}^{p}(\Omega)$ has distributional first derivatives in $\Omega, u$ is a solution to the scalar part of A-Dirac system (1.6) under natural growth condition in $\Omega \backslash E$, and $u$ is of $p$, $k$-oscillation in $\Omega \backslash E$. If for each compact subset $K$ of $E$,

$$
\int_{K(1) \backslash K} d(x, K)^{p(k-1)-k}<\infty
$$

then $u$ extends to a solution of the A-Dirac equation in $\Omega$.

\section{A-Dirac operator and correspondence definition}

In this section, we introduce an $A$-Dirac system. In order to definite the $A$-Dirac operator, we present the definitions and notations of Clifford algebra at first [3].

We write $\mathcal{U}_{n}$ for the real universal Clifford algebra over $\mathbb{R}^{n}$. The Clifford algebra is generated over $R$ by the basis of reduced products

$$
\left\{e_{1}, e_{2}, \ldots, e_{1} e_{2}, \ldots, e_{1} \cdots e_{n}\right\}
$$

where $\left\{e_{1}, e_{2}, \ldots, e_{n}\right\}$ is an orthonormal basis of $\mathbb{R}^{n}$ with the relation $e_{i} e_{j}+e_{j} e_{i}=-2 \delta_{i j}$. We write $e_{0}$ for the identity. The dimension of $\mathcal{U}_{n}$ is $\mathbb{R}^{2^{n}}$. We have an increasing tower $\mathbb{R} \subset$ 
$\mathbb{C} \subset \mathbb{H} \subset \mathcal{U} \subset \cdots$. The Clifford algebra $\mathcal{U}_{n}$ is graded algebra as $\mathcal{U}_{n}=\bigoplus_{l} \mathcal{U}_{n}^{l}$, where $\mathcal{U}_{n}^{l}$ are those elements whose reduced Clifford products have length $l$.

For $A \in \mathcal{U}_{n}, \operatorname{Sc}(A)$ denotes the scalar part of $A$, that is, the coefficient of the element $e_{0}$. Throughout, $\Omega \subset \mathbb{R}$ is a connected and open set with boundary $\partial \Omega$. A Clifford-valued function $u: \Omega \rightarrow \mathcal{U}_{n}$ can be written as $u=\sum_{\alpha} u_{\alpha} e_{\alpha}$, where each $u_{\alpha}$ is real-valued and $e_{\alpha}$ are reduced products. The norm used here is given by $\left|\sum_{\alpha} u_{\alpha} e_{\alpha}\right|=\left(\sum_{\alpha} u_{\alpha}^{2}\right)^{\frac{1}{2}}$. This norm is sub-multiplicative, $|A B| \leq C|A||B|$.

The Dirac operator used here is

$$
D=\sum_{j=1}^{n} e_{j} \frac{\partial}{\partial x_{j}}
$$

Also, $D^{2}=-\triangle$. Here $\triangle$ is the Laplace operator which operates only on coefficients. A function is monogenic when $D u=0$.

Throughout, $Q$ is a cube in $\Omega$ with volume $|Q|$. We write $\sigma Q$ for the cube with the same center as $Q$ and with side length $\sigma$ times that of $Q$. For $q>0$, we write $L^{q}\left(\Omega, \mathcal{U}_{n}\right)$ for the space of Clifford-valued functions in $\Omega$ whose coefficients belong to the usual $L^{q}(\Omega)$ space. Also, $W^{1, p}\left(\Omega, \mathcal{U}_{n}\right)$ is the space of Clifford-valued functions in $\Omega$ whose coefficients as well as their first distributional derivatives are in $L^{q}(\Omega)$. We also write $L_{\text {loc }}^{q}\left(\Omega, \mathcal{U}_{n}\right)$ for $\cap L^{q}\left(\Omega^{\prime}, \mathcal{U}_{n}\right)$, where the intersection is over all $\Omega^{\prime}$ compactly contained in $\Omega$. We similarly write $W_{\text {loc }}^{1, p}\left(\Omega, \mathcal{U}_{n}\right)$. Moreover, we write $\mathcal{M}_{\Omega}=\left\{u: \Omega \rightarrow \mathcal{U}_{n} \mid D u=0\right\}$ for the space of monogenic functions in $\Omega$.

Furthermore, we define the Dirac-Sobolev space

$$
W^{D, p}(\Omega)=\left\{\left.u \in \mathcal{U}_{n}\left|\int_{\Omega}\right| u\right|^{p}+\int_{\Omega}|D u|^{p}<\infty\right\} .
$$

The local space $W_{\text {loc }}^{D, p}$ is similarly defined. Notice that if $u$ is monogenic, then $u \in L^{p}(\Omega)$ if and only if $u \in W^{D, p}(\Omega)$. Also, it is immediate that $W^{1, p}(\Omega) \subset W^{D, p}(\Omega)$.

Under such definitions and notations, we can introduce the operator of $A$-Dirac. Define the linear isomorphism $\theta: \mathbb{R}^{n} \rightarrow \mathcal{U}_{n}^{1}$ by

$$
\theta\left(\omega_{1}, \ldots, \omega_{n}\right)=\sum_{i=1}^{n} \omega_{i} e_{i}
$$

For $x, y \in \mathbb{R}^{n}$, with $D u$ defined by $\theta(\nabla \phi)=D \phi$ for a real-valued function $\phi$, we have

$$
\begin{aligned}
& -\operatorname{Re}(\theta(x) \theta(y))=\langle x, y\rangle, \\
& |\theta(x)|=|x| .
\end{aligned}
$$

Here, $\tilde{A}(x, \xi): \Omega \times \mathcal{U}_{1} \rightarrow \mathcal{U}_{1}$ is defined by

$$
\tilde{A}(x, \xi)=\theta A\left(x, \theta^{-1} \xi\right)
$$

which means that (1.5) is equivalent to

$$
\int_{\Omega} \operatorname{Re}(\theta A(x, \nabla u) \theta(\nabla \phi)) d x=\int_{\Omega} \operatorname{Re}(\tilde{A}(x, D u) D \phi) d x=\int_{\Omega} \operatorname{Re}(f(x, D u) \theta \phi) d x .
$$


For the Clifford conjugation $\overline{\left(e_{j 1} \cdots e_{j l}\right)}=(-1)^{l} e_{j l} \cdots e_{j 1}$, we define a Clifford-valued inner product as $\bar{\alpha} \beta$. Moreover, the scalar part of this Clifford inner product $\operatorname{Re}(\bar{\alpha} \beta)$ is the usual inner product in $\mathbb{R}^{2^{n}},\langle\alpha, \beta\rangle$, when $\alpha$ and $\beta$ are identified as vectors.

For convenience, we replace $\tilde{A}$ with $A$ and recast the structure equations above and define the operators:

$$
A(x, \xi): \Omega \times \mathcal{U}_{n} \rightarrow \mathcal{U}_{n}
$$

where $A$ preserves the grading of the Clifford algebra, $x \rightarrow A(x, \xi)$ is measurable for all $\xi$, and $\xi \rightarrow A(x, \xi)$ is continuous for a.e. $x \in \Omega$. Furthermore, here $A(x, \xi)$ satisfies the structure conditions with $p>1$ :

$$
\begin{aligned}
& \operatorname{Re}(\overline{A(x, \xi) \xi}) \geq \lambda|\xi|^{p} \\
& |A(x, \xi)| \leq a|\xi|^{p-1}
\end{aligned}
$$

for some constant $a>0$. Hence we can definite the weak solution of equation (1.6) as follows.

Definition 2.1 A Clifford-valued function $u \in W_{\text {loc }}^{D \cdot p}\left(\Omega, \mathcal{U}_{n}^{k}\right)$, for $k=0,1, \ldots, n$, is a weak solution to equation (1.6) under structure conditions (2.10) and (2.11), and further assume that the inhomogeneity term $f(x, D u)$ satisfies the natural growth condition

$$
f(x, D u) \leq a_{1}|D u|^{p}+a_{2}, \quad|u|<M<+\infty \text { and } \lambda>a_{1} M
$$

for certain constants $a_{1}$ and $a_{2}$ and $\lambda>0$. Then for all $\phi \in W^{1, p}\left(\Omega, \mathcal{U}_{n}^{k}\right)$ with compact support, we have

$$
\int_{\Omega} \overline{A(x, D u)} D \phi d x=\int_{\Omega} \overline{f(x, D u)} \phi d x
$$

Notice that when $A$ is the identity, then the homogeneity part of (2.13),

$$
\int_{\Omega} \overline{A(x, D u)} D \phi d x=0
$$

is the Clifford Laplacian. Moreover, these equations generalize the important case of the $P$-Dirac equation

$$
D\left(|D u|^{n-2} D u\right)=0 .
$$

Here, $A(x, \xi)=|\xi|^{p-2} \xi$.

These equations were introduced and their conformal invariance was studied in [2].

Furthermore, when $u$ is a real-valued function, (2.14) implies that $A(x, \nabla u)$ is a harmonic field and locally there exists a harmonic function $H$ such that $A(x, \nabla u)=\nabla H$. If $A(x, \xi)$ is invertible, then $\nabla u=A^{-1}(x, \nabla H)$. Hence regularity of $A$ implies regularity of the solution $u$.

In general, $A$-harmonic functions do not have such regularity. This suggests the study of the scalar part of system equation (2.13) in general. Thus, a Caccioppoli estimate for solutions to the scalar part of (2.13) is necessary. This is the topic of the next section. 


\section{The proof of main results}

In this section, we establish the main results. At first, a suitable Caccioppoli estimate for solutions to (2.13) is necessary. Just as which appears in $[3,4]$.

Theorem 3.1 Let $u$ be a solution to the scalar part of (2.13) defined by Definition 2.1, and $Q$ is a cube in $\Omega$. Then, for $\sigma>1$, we have

$$
\int_{Q}|D u|^{p} d x \leq C|Q|^{-\frac{p}{n}} \int_{\sigma Q}\left|u-u_{\sigma Q}\right|^{p} d x+C|Q|^{\frac{n(p-1)+p}{n(p-1)}}
$$

Proof Let the cut-off function $\eta \in C_{0}^{\infty}(\Omega), \eta>0, \eta=1$ in $Q$ and $|D \eta| \leq C|Q|^{-1 / n}$. Choose $\phi=\left(u-u_{\sigma Q}\right) \eta^{p}$ as a test function in (2.13). Then

$$
D \phi=p \eta^{p-1}(D \eta)\left(u-u_{\sigma Q}\right)+\eta^{p} D u .
$$

The solution definition (2.13) yields

$$
\begin{aligned}
& \int_{\sigma Q} \overline{A(x, D u)} p \eta^{p-1}(D \eta)\left(u-u_{\sigma Q}\right) d x+\int_{\sigma Q} \overline{A(x, D u)} \eta^{p} D u d x \\
& =\int_{\sigma Q} \overline{f(x, D u)}\left(u-u_{\sigma Q}\right) \eta^{p} d x .
\end{aligned}
$$

Using the structure condition (2.10), we have

$$
\begin{aligned}
& \lambda \int_{\sigma Q}|D u|^{p} \eta^{p} d x \\
& \quad \leq \int_{\sigma Q} \overline{f(x, D u)}\left(u-u_{\sigma Q}\right) \eta^{p} d x+\left[\int_{\sigma Q} \overline{A(x, D u)} p \eta^{p-1}(D \eta)\left(u-u_{\sigma Q}\right) d x\right] \\
& \quad=I_{1}+I_{2} .
\end{aligned}
$$

By natural growth condition (2.12) and then using Young's inequality, we have

$$
\begin{aligned}
I_{1} & \leq \int_{\sigma Q} a_{1}|D u|^{p}\left(u-u_{\sigma Q}\right) \eta^{p} d x+\int_{\sigma Q} a_{2} \eta^{p}\left(u-u_{\sigma Q}\right) d x \\
& \leq a_{1} M \int_{\sigma Q}|D u|^{p} \eta^{p} d x+\int_{\sigma Q} \frac{u-u_{\sigma Q}}{|Q|^{\frac{1}{n}}}\left(a_{2}|Q|^{\frac{1}{n}} \eta^{p}\right) d x \\
& \leq a_{1} M \int_{\sigma Q}|D u|^{p} \eta^{p} d x+\varepsilon_{1} \int_{\sigma Q} \frac{\left(u-u_{\sigma Q}\right)^{p}}{|Q|^{\frac{p}{n}}} d x+C\left(\varepsilon_{1}\right) \int_{\sigma Q}\left(a_{2}|Q|^{\frac{1}{n}} \eta^{p}\right)^{\frac{p}{p-1}} d x .
\end{aligned}
$$

Notice that

$$
\int_{\sigma Q}\left(a_{2}|Q|^{\frac{1}{n}} \eta^{p}\right)^{\frac{p}{p-1}} d x \leq C_{1}|Q|^{\frac{n(p-1)+p}{n(p-1)}} .
$$

Then, using (2.11), Hölder's and then Young's inequalities, we have

$$
\begin{aligned}
\left|I_{2}\right| & \leq p a \int_{\sigma Q}\left|u-u_{\sigma Q}\right||\nabla \eta||D u|^{p-1}|\eta|^{p-1} d x \\
& \leq C\left(\int_{\sigma Q}\left|u-u_{\sigma Q}\right|^{p}|\nabla \eta|^{p} d x\right)^{\frac{1}{p}}\left(\int_{\sigma Q}|D u|^{p} \eta^{p} d x\right)^{\frac{p-1}{p}}
\end{aligned}
$$




$$
\begin{aligned}
& \leq C\left(\varepsilon_{2}\right)\left(\int_{\sigma Q}\left|u-u_{\sigma Q}\right|^{p}|\nabla \eta|^{p} d x\right)^{\frac{1}{p} \cdot p}+\varepsilon_{2} \int_{\sigma Q}|D u|^{p} \eta^{p} d x \\
& \leq C_{2} \int_{\sigma Q}\left|u-u_{\sigma Q}\right|^{p}|Q|^{-\frac{p}{n}} d x+\varepsilon_{2} \int_{\sigma Q}|D u|^{p} \eta^{p} d x
\end{aligned}
$$

Combining (3.4), (3.5) with (3.6) in inequality (3.3), we obtain

$$
\begin{aligned}
(\lambda & \left.-a_{1} M-\varepsilon_{2}\right) \int_{\Omega}|D u|^{p}|\eta|^{p} d x \\
& \leq\left(C_{2}+C\left(\varepsilon_{1}\right)\right)|Q|^{-\frac{p}{n}} \int_{\sigma Q}\left|u-u_{\sigma Q}\right|^{p} d x+\varepsilon_{1} C_{1}|Q|^{\frac{n(p-1)+p}{n(p-1)}} .
\end{aligned}
$$

Choosing $\varepsilon_{2}$ small enough such that $\lambda-a_{1} M-\varepsilon_{2}>0$, we have

$$
\int_{Q}|D u|^{p} d x \leq C|Q|^{-\frac{p}{n}} \int_{\sigma Q}\left|u-u_{\sigma Q}\right|^{p} d x+C|Q|^{\frac{n(p-1)+p}{n(p-1)}}
$$

This completes the proof of Theorem 3.1.

In order to remove singularity of solutions to the $A$-Dirac system, we also need the fact that real-valued functions satisfy various regularity properties. Thus we have Definition 5.1 [3].

Definition 3.2 Assume that $u \in L_{\text {loc }}^{1}\left(\Omega, \mathcal{U}_{n}\right), q>0$ and that $-\infty<k<1$. We say that $u$ is of $q$, $k$-oscillation in $\Omega$ when

$$
\sup _{2 Q \subset \Omega}|Q|^{-(q k+n) / q n} \inf _{u_{Q} \in \mathcal{M}_{Q}}\left(\int_{Q}\left|u-u_{Q}\right|^{q}\right)^{1 / q}<\infty .
$$

The infimum over monogenic functions is natural since they are trivially solutions to an $A$-Dirac equation just as constants are solutions to an $A$-harmonic equation. If $u$ is a function and $q=1$, then (3.7) is equivalent to the usual definition of the bounded mean oscillation when $k=0$ and (3.7) is equivalent to the usual local Lipschitz condition when $0<k \leq 1$ [13]. Moreover, at least when $u$ is a solution to an $A$-harmonic equation, (3.7) is equivalent to a local order of growth condition when $-\infty<k<1[4,14]$. In these cases, the supremum is finite if we choose $u_{\mathrm{Q}}$ to be the average value of the function $u$ over the cube $Q$. It is easy to see that in condition (3.7) the expansion factor ' 2 ' can be replaced by any factor greater than ' 1 '.

If the coefficients of an $A$-Dirac solution $u$ are of bounded mean oscillation, local Hölder continuous, or of a certain local order of growth, then $u$ is in an appropriate oscillation class [4].

Notice that monogenic functions satisfy (3.7) just as the space $\backslash$ of constants is a subspace of the bounded mean oscillation and Lipschitz spaces of real-valued functions.

We remark that it follows from Hölder's inequality that if $s \leq q$ and if $u$ is of $q, k$ oscillation, then $u$ is of $s, k$-oscillation.

The following lemma [3] shows that Definition 3.2 is independent of the expansion factor of the sphere. 
Lemma 3.3 Suppose that $F \in L_{\mathrm{loc}}^{1}(\Omega, \mathbb{R}), F>0$ a.e., $r \in \mathbb{R}$ and $\sigma_{1}, \sigma_{2}>1$. If

$$
\sup _{\sigma_{1} Q \subset Q}|Q|^{r} \int_{Q} F d x<\infty
$$

then

$$
\sup _{\sigma_{2} Q \subset Q}|Q|^{r} \int_{Q} F d x<\infty .
$$

Then we proceed to prove the main result, Theorem 1.2.

Proof of Theorem 1.2 Let $Q$ be a cube in the Whitney decomposition of $\Omega \backslash E$. The decomposition consists of closed dyadic cubes with disjoint interiors which satisfy

(a) $\Omega \backslash E=\bigcup_{Q \in \mathcal{W}} Q$,

(b) $|Q|^{1 / n} \leq d(Q, \partial \Omega) \leq 4|Q|^{1 / n}$,

(c) $(1 / 4)\left|Q_{1}\right|^{1 / n} \leq\left|Q_{2}\right|^{1 / n} \leq 4\left|Q_{1}\right|^{1 / n}$ when $Q_{1} \cap Q_{2}$ is not empty.

Here, $d(Q, \partial \Omega)$ is the Euclidean distance between $Q$ and the boundary of $\Omega$ [15].

If $A \subset \mathbb{R}^{n}$ and $r>0$, then we define the $r$-inflation of $A$ as

$$
A(r)=\bigcup B(x, r) .
$$

Using the Caccioppoli estimate and the $p, k$-oscillation condition, we have

$$
\begin{aligned}
\int_{Q}|D u|^{p} d x & \leq C|Q|^{-\frac{p}{n}} \int_{\sigma Q}\left|u-u_{\sigma Q}\right|^{p} d x+C|Q|^{\frac{n(p-1)+p}{n(p-1)}} \\
& \leq C \inf _{u_{Q} \in \mathcal{M}_{\sigma Q}}|Q|^{-\frac{p}{n}} \int_{\sigma Q}\left|u-u_{\sigma Q}\right|^{p} d x+C|Q|^{\frac{n(p-1)+p}{n(p-1)}} \\
& \leq C|Q|^{-\frac{p}{n}} \cdot|Q|^{\frac{p k+n}{n}}+C|Q|^{\frac{n(p-1)+p}{n(p-1)}} \leq C|Q|^{a} .
\end{aligned}
$$

Here, $a=(n+p k-p) / n$ and note that $-\infty<k \leq 1$. Since the problem is local (use a partition of unity), we show that (2.13) holds whenever $\phi \in W_{0}^{1, p}\left(B\left(x_{0}, r\right)\right)$ with $x_{0} \in E$ and $r>0$ sufficiently small. Choose $r=(1 / 5 \sqrt{n}) \min \left\{1, d\left(x_{0}, \partial \Omega\right)\right\}$, and let $K=E \cap \bar{B}\left(x_{0}, 4 r\right)$. Then $K$ is a compact subset of $E$. Also, let $W_{0}$ be those cubes in the Whitney decomposition of $\Omega \backslash E$ which meet $B=B\left(x_{0}, r\right)$. Notice that each cube $Q \in W_{0}$ lies in $K(1) \backslash K$. Let $\gamma=p(k-1)-k$. First, since $\gamma \geq-1$, from [2] we have $m(K)=m(E)=0$. Also, since $a-n \geq \gamma$, using (3.6) and (3.11), we obtain

$$
\begin{aligned}
\int_{B\left(x_{0}, r\right)}|D u|^{p} d x & \leq C \sum_{Q \in W_{0}}|Q|^{a / n} \leq C \sum_{Q \in W_{0}} d(Q, K)^{a} \\
& \leq C \sum_{Q \in W_{0}} \int_{Q} d(x, K)^{a-n} d x \leq C \int_{K(1) \backslash K} d(x, K)^{a-n} d x \\
& \leq C \int_{K(1) \backslash K} d(x, K)^{\gamma} d x<\infty .
\end{aligned}
$$

Hence $u \in W_{\text {loc }}^{D, p}(\Omega)$.

Next, let $B=B\left(x_{0}, r\right)$ and assume that $\psi \in C_{0}^{\infty}(B)$. Also, let $W_{j}, j=1,2, \ldots$, be those cubes $Q \in W_{0}$ with $l(Q) \leq 2^{-j}$. 
Consider the scalar functions

$$
\phi_{j}=\max \left\{\left(2^{-j}-d(x, K)\right) 2^{j}, 0\right\} .
$$

Thus each $\phi_{j}, j=1,2, \ldots$, is Lipschitz, equal to 1 on $K$ and as such $\psi\left(1-\phi_{j}\right) \in W^{1, p}(B \backslash E)$ with compact support. Hence

$$
\begin{aligned}
& \int_{B}[\overline{A(x, D u)} D \psi-\overline{f(x, D u)} \psi] d x \\
& =\int_{B \backslash E}\left[\overline{A(x, D u)} D\left(\psi\left(1-\phi_{j}\right)\right)-\overline{f(x, D u)} \psi\left(1-\phi_{j}\right)\right] d x \\
& \quad+\int_{B}\left[\overline{A(x, D u)} D\left(\psi \phi_{j}\right)-\overline{f(x, D u)} \psi \phi_{j}\right] d x .
\end{aligned}
$$

Let

$$
\begin{aligned}
& J_{1}=\int_{B \backslash E}\left[\overline{A(x, D u)} D\left(\psi\left(1-\phi_{j}\right)\right)-\overline{f(x, D u)} \psi\left(1-\phi_{j}\right)\right] d x, \\
& J_{2}=\int_{B}\left[\overline{A(x, D u)} D\left(\psi \phi_{j}\right)-\overline{f(x, D u)} \psi \phi_{j}\right] d x .
\end{aligned}
$$

Since $u$ is a solution in $B \backslash E, J_{1}=0$.

Also, we have

$$
\begin{aligned}
J_{2} & =\int_{B} A(x, D u) \psi D \phi_{j} d x+\int_{B} \phi_{j} A(x, D u) D \psi d x-\int_{B} \overline{f(x, D u)} \psi \phi_{j} d x \\
& =J_{2}^{\prime}+J_{2}^{\prime \prime}+J_{2}^{\prime \prime \prime} .
\end{aligned}
$$

Now there exists a constant $C$ such that $|\psi| \leq C<\infty$. Hence, using Hölder's inequality,

$$
\begin{aligned}
\left|J_{2}^{\prime}\right| & \leq C \sum_{Q \in W_{j}} \int_{Q}|A(X, D u)|\left|D \phi_{j}\right| d x \\
& \leq C \sum_{Q \in W_{j}} \int_{Q}|D u|^{p-1}\left|D \phi_{j}\right| d x \\
& \leq C \sum_{Q \in W_{j}}\left(\int_{Q}|D u|^{p} d x\right)^{(p-1) / p}\left(\int_{Q}\left|D \phi_{j}\right|^{p} d x\right)^{1 / p} .
\end{aligned}
$$

Next, using the estimate (3.11), the above becomes

$$
\left|J_{2}^{\prime}\right| \leq C \sum_{Q \in W_{j}}|Q|^{(p(k-1)+n)(p-1) / n p^{j}}|Q|^{1 / p} .
$$

Now, for $x \in Q \in W_{j}, d(x, K)$ is bounded above and below by a multiple of $|Q|^{1 / n}$ and for $Q \in W_{j},|Q|^{1 / n} \leq 2^{-j}$. Hence

$$
\left|J_{2}^{\prime}\right| \leq C \sum_{Q \in W_{j}}|Q|^{-1 / n+1 / p+(p(k-1)+n)(p-1) / n p} \leq C \int_{\bigcup W_{j}} d(x, K)^{p(k-1)-k} d x
$$

Since $\bigcup W_{j} \subset K(1) \backslash K$ and $\left|\bigcup W_{j}\right| \rightarrow 0$ as $j \rightarrow \infty$, it follows that $J_{2}^{\prime} \rightarrow 0$ as $j \rightarrow \infty$. 
Next, again using Hölder's inequality, we obtain

$$
\begin{aligned}
\left|J_{2}^{\prime \prime}\right| & \leq \sup _{B}|D \psi|\left(\int_{\bigcup W_{j}}|D u|^{p} d x\right)^{(p-1) / p}\left|\bigcup W_{j}\right|^{1 / p} \\
& \leq C\left(\int_{K \backslash K(1)}|D u|^{p} d x\right)^{(p-1) / p}\left|\bigcup W_{j}\right|^{1 / p} .
\end{aligned}
$$

Since $u \in W_{\text {loc }}^{1, D}(\Omega)$ and $\left|\bigcup W_{j}\right| \rightarrow 0$ as $j \rightarrow \infty$, we have that $J_{2}^{\prime \prime} \rightarrow 0$ as $j \rightarrow \infty$. Hence $J_{2} \rightarrow 0$.

In order to estimate $J_{2}^{\prime \prime \prime}$, using (3.12), we have

$$
J_{2}^{\prime \prime \prime}=\int_{B} \overline{f(x, D u)} \psi \phi_{j} d x \leq C \int_{B}|D u|^{p} d x+C \int_{B} d x=J_{3}^{\prime}+J_{3}^{\prime \prime} .
$$

Similar as the estimate of (3.18), using the Caccioppoli inequality and inequality (3.11), we get

$$
\begin{aligned}
J_{3}^{\prime} & \leq C \sum_{Q \in W_{j}} \int_{Q}|D u|^{p} d x \leq C \sum_{Q \in W_{j}}|Q|^{(n+p k-p) / n} \\
& \leq C \int_{\cup W_{j}} d(x, K)^{n+p k-p} d x \leq C \int_{\cup W_{j}} d(x, K)^{p(k-1)-k} d x . \\
& \rightarrow 0 \quad(j \rightarrow \infty)
\end{aligned}
$$

and

$$
\begin{aligned}
J_{3}^{\prime \prime} & \leq C \sum_{Q \in W_{j}} \int_{Q} d x=C \sum_{Q \in W_{j}}|Q| \\
& \leq C \int_{\cup W_{j}} d(x, K)^{n} d x \leq C \int_{\cup W_{j}} d(x, K)^{n+p k-p} d x \\
& \leq C \int_{\cup W_{j}} d(x, K)^{p(k-1)-k} d x \\
& \rightarrow 0 \quad(j \rightarrow \infty) .
\end{aligned}
$$

Hence $J_{3} \rightarrow 0$.

Combining the estimates $J_{1}$ and $J_{2}$ in equation (3.14), we prove Theorem 1.2.

Competing interests

The authors declare that they have no competing interests.

\section{Authors' contributions}

FS participated in design of the study and drafted the manuscript. SC participated in conceived of the study and the amendment of the paper. All authors read and approved the final manuscript.

\section{Acknowledgements}

This work was supported by the National Natural Science Foundation of China (No. 11201415), Natural Science Foundation of Fujian Province (2012J01027) and Training Programme Foundation for Excellent Youth Researching Talents of Fujian's Universities (JA12205). 


\section{References}

1. Abreu-Blaya, R, Bory-Reyes, J, Peña-Peña, D: Jump problem and removable singularities for monogenic functions. J. Geom. Anal. 17(1), 1-13 (2007)

2. Nolder, CA, Ryan, J: p-Dirac operators. Adv. Appl. Clifford Algebras 19(2), 391-402 (2009)

3. Nolder, CA: A-Harmonic equations and the Dirac operator. J. Inequal. Appl. 2010, Article ID 124018 (2010)

4. Nolder, CA: Nonlinear A-Dirac equation. Adv. Appl. Clifford Algebras 21(2), 429-440 (2011)

5. Wang, C: A remark on nonlinear Dirac equations. Proc. Am. Math. Soc. 138(10), 3753-3758 (2010)

6. Wang, C, Xu, D: Remark on Dirac harmonic maps. Int. Math. Res. Not. 20, 3759-3792 (2009)

7. Chen, Q, Jost, J, Li, J, Wang, G: Dirac-harmonic maps. Math. Z. 254(2), 409-432 (2006)

8. Chen, Q, Jost, J, Li, J, Wang, G: Regularity theorems and energy identities for Dirac-harmonic maps. Math. Z. 251(1), 61-84 (2005)

9. Chen, Q, Jost, J, Wang, G: Nonlinear Dirac equations on Riemann surfaces. Ann. Glob. Anal. Geom. 33(3), 253-270 (2008)

10. Kaufman, R, Wu, JM: Removable singularities for analytic or subharmonic functions. Ark. Mat. 18(1), 107-116 (1980)

11. Koskela, P, Martio, O: Removability theorems for solutions of degenerate elliptic partial differential equations. Ark. Mat. 31(2), 339-353 (1993)

12. Kilpelainen, $T$, Zhong, $X$ : Removable sets for continuous solutions of quasilinear elliptic equations. Proc. Am. Math. Soc. 130(6), 1681-1688 (2002)

13. Meyers, NG: Mean oscillation over cubes and Hölder continuity. Proc. Am. Math. Soc. 15(5), 717-721 (1964)

14. Langmeyer, N: The quasihyperbolic metric, growth, and John domains. Ann. Acad. Sci. Fenn. Math. 23(1), 205-224 (1998)

15. Stein, EM: Singular Integrals and Differentiablity Properties of Functions. Princeton Mathematical Series, vol. 30. Princeton University Press, Princeton (1970)

10.1186/1029-242X-2013-463

Cite this article as: Sun and Chen: A-Harmonic operator in the Dirac system. Journal of Inequalities and Applications 2013, 2013:463

\section{Submit your manuscript to a SpringerOpen ${ }^{0}$ journal and benefit from:}

- Convenient online submission

- Rigorous peer review

Immediate publication on acceptance

- Open access: articles freely available online

- High visibility within the field

- Retaining the copyright to your article 